ataxia was made. He subsequently developed progressive scoliosis, pyramidal weakness and increasing ataxia and is now wheelchair-bound.

Six months before referral he had suffered an isolated episode of acute chest pain and loss of consciousness. A cardiac dysrhythmia was suspected but his ECG showed no change from earlier recordings. In May 1984 he began to experience fainting episodes at school and 6 months later presented as an emergency with acute chest pain and dyspnoea and was found to have a paroxysmal supraventricular tachycardia. This was treated with intravenous practolol. The resting ECG now showed the typical features of WPW Type B (fig). Treatment with propranolol and quinidine has prevented further symptoms to date.

Cardiac symptoms and signs occur in about one-third of patients with Friedreich's $\operatorname{ataxia}^{1}$ but electrocardiographic abnormalities are more common, ${ }^{2}$ with $\mathrm{T}$-wave inversion in several leads, with or without other features of left ventricular hypertrophy, being reported in $30 \%^{3}$ to $90 \%{ }^{4}$ of cases in different series. In a series of 115 cases, Harding and Hewer ${ }^{2}$ found only one patient with a short PR interval and none with atrio-ventricular block. Supraventricular tachycardia was noted in eight cases and ventricular extrasystoles in two. Atrial fibrillation occurred in one patient;

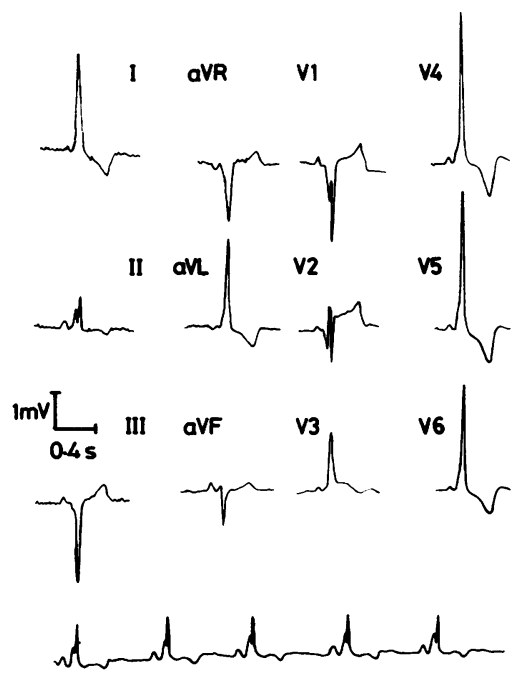

Fig Electrocardiogram recorded in November 1984, showing left ventricular hypertrophy and WPW Type B. The PR interval is $0.04 \mathrm{~ms}$, delta waves are present and the QRS complex is widened $(0.12 \mathrm{~ms})$ with a negative initial deflection in $V_{1}$. Widespread T-wave inversion is evident. both arrhythmias were associated with a poor prognosis. ${ }^{25}$

While there is often evidence of asymptomatic cardiac involvement at the onset of neurological symptoms, the development of cardiac signs and symptoms prior to neurological dysfunction is rare. ${ }^{26-8}$ Berg et al $^{6}$ described two sibs who were eventually found to have Friedreich's ataxia, who presented with cardiac failure at 3 and 5 years of age, and Nadas $\mathrm{et} \mathrm{al}^{7}$ reported a $31 / 2$ year old child who had cardiomegaly, a murmur and ECG abnormalities at the time of presentation with unsteadiness. However, onset under one year of age as in the present case has not been documented previously.

Pre-excitation syndromes such as WPW are generally believed to be due to the presence of accessory functioning pathways between the atria and ventricles. In typical cases of WPW these accessory pathways, which are composed of functional myocardial cells (or rarely specialised conducting tissues), are believed to be vestiges of a more extensive atrioventricular ring found in the embryonic heart. ${ }^{9}$ However, the present case illustrates that such pathways are not necessarily congenital but can evolve as a result of a progressive cardiomyopathy. Our patient had a form of hypertrophic cardiomyopathy and this appears to be the commonest cardiac disorder in Friedreich's ataxia. ${ }^{1}$ Concentric left ventricular hypertrophy occurs most frequently ${ }^{1}$ but outflow tract obstruction due to asymmetric septal hypertrophy can develop and was demonstrated in nearly one-third of cases in one series; ${ }^{10}$ cardiac dysrhythmias, including WPW, are not uncommon under such circumstances. $^{11}$

R NEIL BALDWIN
RUSSELL JM LANE
Associated Unit of Neurological Science,
Walton Hospital,
Rice Lane,
Liverpool L9 IAE,UK

References

1 Harding AE. Friedreich's Ataxia. In: The Hereditary Ataxias and Related Disorders. London: Churchill Livingstone, 1984: 57-103.

2 Harding AE, Hewer RL. The heart disease of Friedreich's ataxia: a clinical and electrocardiographic study of 115 patients with an analysis of serial electrocardiographic changes in 30 cases. $Q J$ Med 1983;52:489-502.

3 Evans W, Wright G. The electrocardiogram in Friedreich's disease. Br Heart J 1942;4:91-9.
4 Malo S, Latour Y, Cote M, Goeffrey G, Lemieux B, Barbeau A. Electrocardiographic and vectocardiographic findings in Friedreich's ataxia. Can J Neurol Sci 1976;3:323-8.

5 Hewer RL. Study of fatal cases of Friedreich's ataxia. Br Med J 1968;3:649-52.

6 Berg RA, Kaplan AM, Jarrett PB, Molthan ME. Friedreich's ataxia with acute cardiomyopathy. Am J Dis Child 1980;134:390-3.

7 Nadas AS, Alimurung MM, Sieracki LA. Cardiac manifestations of Friedreich's ataxia. $N$ Eng J Med 1951;244:293-8.

8 Thilenius OG, Grossman BJ. Friedreich's ataxia with heart disease in children. Pediatrics 1961;27:246-54.

9 Becker AE, Bouman LN, Janse MJ, Anderson RH. Functional anatomy of the cardiac conduction system. In: Harrison DC, ed. Cardiac Aryhthmias. A Decade of Progress. Boston: GK Hall, 1981:3-24.

10 Gattiker HF, Davignon A, Bozio A, et al. Electrocardiographic findings in Friedreich's ataxia. Can J Neurol Sci 1976;3:329-32.

11 Behar VS. Asymmetric septal hypertrophy. In: Beeson PB, McDermott W, Wyngaarden JB, eds. Cecil Textbook of Medicine. 15th ed. London: WB Saunders, 1979:1278-9.

Accepted 17 April 1986

Flunitrazepam intoxication simulating structural brainstem lesion

Sir: We wish to report a case of severe brainstem dysfunction provoked by an overdose of flunitrazepam.

A 60-year-old man was brought to the emergency room, unconscious, after being found unresponsive by his wife. He had a past history of depressive illness, but according to his wife his mood had been normal during the previous months. On admission, his blood pressure was $120 / 60 \mathrm{mmHg}$, pulse 68 per minute, temperature $37^{\circ} \mathrm{C}$; his breathing pattern was irregular. General examination revealed no further information. Neurological examination revealed a patient in a deep coma, with no spontaneous limb movements and decerebrate posturing elicited by painful stimulation. He had bilateral unresponsive small pupils. Oculocephalic, oculovestibular, ciliospinal and corneal reflexes were absent. There was an increased tone in lower and upper extremities. Tendon reflexes were brisk and the left plantar response was extensor. There was no nuchal rigidity. Thiamine was given, and the intravenous administration of naloxone and hypertonic glucose did not alter his neurological status. Baseline blood tests, blood gases, chest radiograph, electrocardiogram and CT scan of the head were normal. Elec- 
troencephalogram (EEG) showed a diffuse monorhythmical $9-12 \mathrm{~Hz}$ activity of $50 \mu \mathrm{V}$ amplitude, with no reactivity to painful stimulation. Multi-drug screening tests established intoxication with a benzodiazepine later identified as flunitrazepam.

On the second day the patient gradually regained consciousness. A repeat EEG at that time was normal. After recovery he admitted an attempt at suicide taking 25 tablets each of $2 \mathrm{mg}$ of flunitrazepam.

This case presented two features which have not previously been reported in benzodiazepine-intoxication. Firstly, the asymmetrical neurological signs suggested this patient had a structural brainstem lesion which can not always reliably be excluded by CT scan. This emphasises the necessity of multi-drug screening tests in every patient with "coma of unknown origin" even in the presence of lateralizing signs. Secondly, an alpha coma is relatively rare and has been associated with hypoxic encephalopathy of primary brainstem lesions. ${ }^{1}$ Occasionally however this type of EEG pattern results from an overdose of drugs such as glutethimide, barbiturates and amytriptyline. ${ }^{2}$ Unlike the patients with hypoxic encephalopathy, a drug-induced alpha pattern coma has a good prognosis. ${ }^{3}$ This case demonstrates that flunitrazepam also should be included in the list of drugs that can cause alpha pattern coma.

DIRK DELEU JAAK DE KEYSER Department of Neurology, University Hospital A.Z.-V.U.B. Laarbeeklaan, 101 1090 Brussels, Belgium

\footnotetext{
References

1 Chokroverty S. Alpha-like rhythms in electroencephalograms in coma after cardiac arrest. Neurology 1975;25:655-63.

2 Carroll WM, Mastaglia FL. Alpha and beta coma in drug intoxication uncomplicated by cerebral hypoxia. Electroencephalogr Clin Neurophysiol 1979;46:95-105.

3 Kuriowa Y, Furakawa T, Kuzumoto I. Recovery from drug-induced alpha coma. Neuro$\log y$ 1981;31:1359-61.
}

\section{Distant metastases from a malignant glioma: unusual complications associated with treat- ment of a glioblastoma: distant metastases and focal white matter degeneration}

Sir: Gliomatous metastases have been found in the liver, bone, lung, pleural cavity, pericardium and lymph nodes. ${ }^{1}$ Only rarely do patients develop bone marrow invasion with myelofibrosis and symptomatic anaemia. ${ }^{23}$ We report the second such case in which pancytopenia resulting from marrow invasion was the primary clinical presentation. In addition, white matter degeneration due to the effects of radiotherapy and possible chemotherapy caused a major diagnostic dilemma. The importance of glial fibrillary acid protein staining to confirm the diagnosis of metastatic glioma while the patient is alive is demonstrated.

A 52 year old business executive was well until 1972 when he suffered a generalised seizure. A complete evaluation was negative aside from an EEG which revealed left frontal slowing. He remained neurologically intact aside from occasional right focal motor seizures until 1980 when he developed mild aphasia. A CT scan revealed a contrast enhancing lesion in the left parietal region. Tumour removed by subtotal resection in April 1980 was considered to be a Grade III astrocytoma. The patient continued to worsen and in June 1980, an extensive resection was performed. Between July 18 , 1980 and August 21, 1980 he received 5000 rads whole brain radiotherapy in 25 fractions. A boost of 1000 rads was delivered in five fractions to the left frontal region. Following this the patient received six three day courses of intravenous BCNU given 6 weeks apart from October 1980 to June 1981 totalling $1600 \mathrm{mg} / \mathrm{m}$. Procarbazine was given daily from July 1981 to November 1981 and again from January 1982 to February 1982. He was admitted to the Roger Williams General Hospital on July 2, 1982 because of increasing rightsided weakness and lethargy. Physical examination was remarkable only for a moderate aphasia and a mild right hemiparesis. Laboratory values included: $\mathrm{WBC}$, Hgb. $11 \cdot 8 \%$ mg HCT $32 \cdot 9 \%$, MCV 96, Platelets 197,000 . The blood smear appeared normal. $\mathrm{Fe} / \mathrm{TIBC}=44 / 165$. SMA6, VDRL, PT, APTT and SMA12 were normal aside from: lactate dehydrogenase $418 \mathrm{U} / 1$ (normal $80-210$ ), alkaline phosphatase $438 \mathrm{U} / 1$ (50-260), serum glutamic-oxaloacetic transaminase $31 \mathrm{U} / \mathrm{l}(5-30)$, cholesterol $385 \mathrm{mg} \%$ (160-300). Stool was negative for occult blood. Chest radiographs revealed an abnormal liner density in the right lung base thought to be either scarring or a subsegmental area of atelectasis. The right sixth rib demonstrated a posterolateral fracture and the bones were osteopenic. Contrast enhanced cerebral CT scan revealed bilateral ventricular enlargement, left greater than right, with a semicircular ring enhancing lesion adjacent to the skull in the left superior anterior region and a small left frontal enhancing nodule abutting the ring. He was started on dexamethasone with significant improvement of his intellectual function and to a lesser degree his right sided weakness. Over the next 2 months, the patient's haematological function worsened, with slowly declining platelets and haemoglobin levels. Intermittent transfusions were required to maintain a haematocrit above 20. Alkaline phosphatase continued to be elevated. Chest radiograph was interpreted as showing diffuse bony metastases. Seizure activity became more difficult to control so that carbamazepine was substituted for primidone. Dexamethasone was increased to $6 \mathrm{mg}$ qid because the aphasia and right sided weakness became worse. A repeat CT scan in October 1982 revealed no significant interval change however.

Several attempted bone marrow aspirates and biopsies failed to produce adequate specimens for diagnosis. A biopsy specimen in November 1982 showed a monomorphic proliferation of a malignant cell line of undetermined type. He continued to require transfusion of both blood and platelets. His neurological status continued to deteriorate, although repeat CT scan again did noto reveal any significant change. The patient died in December 1982 from complications $\underset{\mathbb{C}}{\stackrel{P}{C}}$ of pancytopenia and adult respiratory dis- $\varrho$ tress syndrome.

The general necropsy revealed a non- $\bigcirc$ malignant pleural effusion, early pneumoniao and an old myocardial infarction. The spleen revealed small areas of extra-을 medullary haematopoiesis. The vertebrae $\stackrel{\infty}{\rightleftharpoons}$ were infiltrated by a white tissue which on microscopic examination was found to be composed of small undifferentiated tumour cells identical to those noted in the surgical brain specimen of $4 / 22 / 80$. These cells were positively stained with immunoperoxidase for glial fibrillary acid protein. In the brain, a cavitative necrotic lesion of about $4 \mathrm{~cm}$ surrounded by a brownish gliotic zone was found in the left fronto-parietal region. There was a mild yellowish discoloration of the adjacent white matter of the centrum semiovale. The left lateral ventricule was slightly dilated at the frontal end due to gliosis of the adjacent white matter. The cerebellum, pons and medulla showed no significant change. External to the necrotic area was a zone of intense gliosis in which thick-walled blood vessels were seen. Spreading from the edge of the cavity was an area of graded loss of myelinated nerve fibres. ranging from total destruction to a subtle spongiosis of the white matter. There was, however, widespread blood vessel thickening even in areas where there was 